\title{
Cytokines: Role in Human Metabolism
}

\author{
H.P.Sauerwein, T.van der Poll, and J.A. Romijn
}

\section{Introduction}

The metabolic impact of infectious, neoplastic and certain other disease states have long been known to clinicians. Those diseases in general induce, aside specific symptoms associated with the disease itself, general signs and symptoms [1]. These alterations include fever, hypermetabolism, cachexia, leukocytosis, hypoferremia and marked changes in carbohydrate-, protein- and lipid metabolism, resulting in hyperglycemia, sometimes followed by hypoglycemia, increased glucose production, protein catabolism, hypertriglyceridemia, increased free fatty acid (FFA) turnover and decreased ketonemia [2, 3]. It was once believed that these metabolic derangements were induced by the disease itself and that differences in the metabolic response were due to differences between disease states. In recent years however, there has been a growing awareness that the metabolic responses to disease tend to occur in a consistent and predictable manner and that endogenous mediators could be essential elements in the pathogenesis of above-mentioned responses $[1,3,4]$. Some of these mediators, the "classical" hormones are well-known for a longtime: corticosteroids, catecholamines, glucagon, growth-hormone and insulin. The accepted explanation for the changes in glucose-, protein-, and fat metabolism were marked increases in the plasma concentrations of the counter-regulatory hormones together with decreased tissue sensitivity to insulin [2]. These explanations have been substantiated by recent studies [3]. In healthy volunteers was shown that hypermetabolism, hyperglycemia, but not hypoglycemia, protein loss and increased lipolysis can be induced by the infusion of the counter-regulatory hormones. Simultaneous infusion of these hormones was necessary, as separate infusion of each of them provided lesser and sometimes only transient metabolic changes. These metabolic changes however are sometimes also seen despite seemingly normal plasma concentrations of the counter-regulatory hormones [5] whereas other metabolic responses like fever, acute phase protein synthesis, hypoferremia and leukocytosis could be induced only by injection of an inflammatory agent like etiocholanon [3, 4].

Both endocrine and other mediators seem therefore necessary for the complete manifestation of the metabolic response to disease. Recently, considerable interest has been focused on a family of inflammatory mediators called cytokines. These cytokines appear to have metabolic effects related to tissue wasting and therefore may play a role as mediators of the overall response. It is extremely difficult to study the possible metabolic effects of cytokines itself as ex- 
perimental conditions have a major influence on the obtained results and it is even possible that cytokines have no direct effects at all [6]. It is therefore no surprise that until now not many studies have been published on the metabolic effects of cytokines [2]. This chapter will focus on our investigations to develop models for studying acute and chronic metabolic effects of cytokines relevant for the clinical human situation.

\section{Cytokines}

A recent work implicates a class of proteins collectively termed cytokines as important endogenous mediators of the host response. These proteins differ from classic hormones in that cytokines are bioactive at low concentrations (less than $10^{-11} \mathrm{~mol} / \mathrm{l}$ ) and are differentially synthesized at various sites by diverse cells within the body, depending on the location and the nature of the initiating stimulus [7]. The cytokines most widely studied in relation to metabolism are tumor necrosis factor (TNF), interleukin-1 (IL-1) and interleukin-6 (IL-6) [1, 4, 7, 8].

Tumor necrosis factor is produced by monocytes and macrophages, mast cells, endothelial cells and natural killer cells. Provocative stimuli for synthesis are numerous infectious and inflammatory stimuli. Increased levels in plasma are found in patients with very different diseases like parasitic and bacterial infections or malignancy [7].

Interleukin-1 is synthesized predominantly by blood monocytes and tissue macrophages but is also produced by a variety of other cell types like endothelial cells, keratinocytes and neutrophils. It is hardly ever detected in the circulation and is mainly thought to induce its effects by paracrine influences [7]. The mRNA for interleukin- 6 is expressed in numerous tissues like fibroblasts, monocytes, macrophages, keratinocytes and endothelial cells. Its production is enhanced by other cytokines as well as bacterial and viral products. It is easily detectable in the circulation evenly in healthy subjects [7].

The exact metabolic role of each of these cytokines in vivo is not known due to very complex interactions between these mediators itself and between these mediators and the endocrine system. With endotoxin as provocative stimulus, it has been shown in healthy humans that the release of TNF into the circulation peaks after $90 \mathrm{~min}$ which is rapidly followed by the appearance of IL-6 [9]. Apart from triggering cytokine release, endotoxin also elicits a response of the classic counter-regulatory hormones, partly concurrent with the cytokine response $[9,10]$. In vitro TNF induces release of IL-1 and a variety of other mediators [7, 11]. Feedback inhibition of cytokine production also exists as for example, corticosteroids proved to be potent inhibitors of expression of mRNA of both TNF and IL-1 [7].

Due to these complex in vivo interactions between cytokines and the classical hormones, in vitro studies seemed to be more appropriate to unravel the metabolic effects of cytokines itself. However, the conclusions drawn from in vitro experiments proved to be not always transferable to the clinical situation and 
were sometimes even contradictory to in vivo data obtained in metabolic studies. Study design and interspecies differences may be responsible herefore.

Two examples will be given. Hypertriglyceridemia is a well known feature of some infectious diseases. Early experiments showed that TNF is capable of suppressing lipoprotein lipase (LPL) activity in a preadipocyte cell-line [12]. For a long time it was thought that hypertriglyceridemia was due to a decrease in the clearance of triglycerides from the circulation due to this mechanism. Later on, it was shown that TNF indeed impairs LPL activity in adipose tissue in vivo, however it appeared to stimulate the activity of LPL in general other organs, including heart, lung and liver [13]. Additional investigations have revealed that decreased LPL activity does not significantly contribute to hypertriglyceridemia in infection, but that the rise in plasma triglycerides is predominantly caused by increased lipid synthesis by the liver [14].

All these studies were done in rats. The problem of interspecies differences is demonstrated by the fact that TNF does not inhibit lipoprotein lipase activity in cultured human adipocytes [15]. Interspecies differences also exist for IL-1 as it has been shown in vitro that gluconeogenesis is stimulated by IL-1 in the rat [16] inhibited in mice [17], while it has no effect in rabbits in vivo [18]. To circumvent such problems and to obtain clinical relevant data, we have concentrated on studies in humans in our try to unravel the metabolic effects of cytokines. The first data, mainly directed at the development of a good model for studying acute and more chronic effects of cytokines, will be presented.

\section{Human Studies}

\section{Acute Effects of Cytokines}

Several lines of evidence indicate that excessive TNF release into the circulation is the initial and pivotal event in the development of the septic syndrome. This is most strongly emphasized by observations in septic baboons, in which passive immunization against TNF both attenuates the systemic appearance of IL-1 $\beta$ and IL-6, as well as prevents lethality [19]. The metabolic effects of TNF in humans have not been studied extensively. The studies published so far have been done in cancer patients $[20,21]$ which hampers interpretation since cancer patients may be chronically exposed to increased endogenous TNF levels [22] and prolonged exposure to TNF may lead to increased tolerance [23]. Therefore, we performed a cross-over saline controlled study in healthy males studying the effects of a bolus i.v. injection of TNF on several aspects of metabolism [24]. The observation time was $10.5 \mathrm{hr}$.

TNF induced a transient stress hormone response which was associated with rises in glucose turnover (mean increase 10\%), FFA turnover (120\%) and energy expenditure (34\%) and a decrease in glucose oxidation. Both the hormonal and metabolic changes observed after TNF administration were similar to those found in septicemia. Thus, systemic TNF release early in the cause of septicemia may be of importance for the initiation of the metabolic response to acute infection. The septicemia increased levels of cytokines and stress hormones coexist. 
Our study in healthy humans shows that the in vivo administration of cytokines may serve as a proper model to examine the metabolic effects of cytokines in the concomitant presence of enhanced stress hormone release.

Acute administration of cytokines will induce counter-regulatory hormone response. The metabolic effect of cytokines, in absence of counter-regulatory hormone response, could be different from those in acute infection and it was considered useful to find a model for studying cytokine effects in absence of increased secretion of the catabolic hormones.

\section{Chronic Effects of Cytokines}

Hypermetabolism, increased glucose production, glucose disposal, increased lipolysis and hypertriglyceridemia are most prominent in acute, severe infection like sepsis, but have also been reported in mild infection with or without fever [25]. Reports on counter-regulatory hormone concentrations in mild infection are scarce. Stimulation of the secretion of cortisol, growth hormone and glucagon is frequently reported; data on plasma catecholamine concentration are varying, while basal insulin concentration usually is in the normal range [25].

A few reports have been published suggesting that cytokine production (TNF, IL-1, IL-6) is increased in human immune deficiency virus (HIV), infection either reflected as increased plasma levels or increased production by peripheral blood monocytes [25]. Host metabolism has hardly been studied in patients with the acquired immunodeficiency syndrome (AIDS). Wasting and hypertriglyceridemia are the only metabolic alterations in relation to HIV infection reported so far [25]. The same holds true for data on counter-regulatory hormone concentrations in AIDS except for a few conflicting reports on cortisol concentrations [25].

Based on the abovementioned data, we thought that HIV infection could possibly be a useful model to study the influences of cytokines on metabolism in the absence of increased plasma concentration of counter-regulatory hormones. Metabolism and counter-regulatory hormone concentrations were therefore studied in patients with AIDS under strictly controlled circumstances. One of the main entrance criteria was absence of clinically active opportunistic infection for at least two months, to ensure that data on host metabolism would reflect the ef-

Table 1. Classical hormone concentration in HIV infection (mean \pm SD)

\begin{tabular}{lcccccc}
\hline & \multicolumn{2}{c}{ Symptomatic HIV } & \multicolumn{2}{c}{ Asymptomatic HIV } & \multicolumn{2}{c}{ Control } \\
\hline Insulin [mU/l] & 7 & \pm 1 & 8 & \pm 1 & 7 & \pm 1 \\
Glucagon [ng/l] & 109 & \pm 3 & - & & 99 & \pm 15 \\
Epinephrine [ng/l] & 50 & \pm 7 & 62 & \pm 12 & 69 & \pm 8 \\
Norepinephrine [ng/l] & 233 & $\pm 26^{*}$ & 226 & $\pm 30^{*}$ & 367 & \pm 38 \\
Cortisol [micromol/l] & $0.31 \pm 0.04$ & $0.27 \pm 0.04$ & $0.27 \pm 0.03$ \\
Growth hormone [mU/1] & 6 & \pm 2 & 7 & \pm 4 & $<2$ (all) \\
\hline
\end{tabular}

${ }^{*} \mathrm{p}<0.05$ vs control. 
fects of chronic HIV infection only (possibly due to chronic cytokines overproduction), without possibly confounding metabolic impact of simultaneous present acute (opportunistic) infections. The data on classical hormone concentrations in 18 patients with AIDS and 11 asymptomatic HIV infected patients compared to a control group are given in Table 1 . The results clearly show that counter-regulatory hormone response is not stimulated in asymptomatic or symptomatic HIV infection, supporting our idea that HIV infection could be a useful model.

\section{Metabolic Effects in HIV Infection}

Weight loss is frequently found in symptomatic HIV infection even in the absence of anorexia or malabsorption. To investigate whether increased resting energy expenditure might be responsible for weight loss in patients infected with HIV, indirect calorimetry was done in patients with symptomatic HIV infection (CDC IV) free of clinically active opportunistic infection; the data were compared with a group of healthy volunteers with similar food intake, body composition and sex (Table 2). Resting energy expenditure was significantly higher in the patients $(+9 \%)$. Hypermetabolism is therefore a feature of symptomatic HIV infection [26]. In addition, hypermetabolism appears to be a relative early symptom in HIV infection, as even in asymptomatic patients an increase in resting energy expenditure of $8 \%$ was found [27].

The changes in glucose metabolism induced by HIV infection proved to be rather remarkable [28]. After an overnight fast, endogenous glucose production $(2.52 \pm 0.07 \mathrm{mg} / \mathrm{kg} / \mathrm{min})$ was not different from the value in healthy subjects $(2.24 \pm 0.17 \mathrm{mg} / \mathrm{kg} / \mathrm{min})$ but plasma glucose concentration was significantly lower indicating increased peripheral glucose clearance. This was confirmed by a two-step euglycemic insulin clamp. HIV infection is therefore characterized by increased insulin sensitivity, while infectious diseases in general induce insulin resistance [25]. Another remarkable feature was the influence of HIV infection in insulin clearance. During insulin infusion, significantly lower values for plasma insulin concentrations were obtained compared to values found in control subjects [26]. Increased insulin clearance is an usual finding in many diseases but in general is accompanied by a decrease in insulin effect while in HIV infection an increase in insulin effect is found [25].

Table 2. Impact of HIV infection and other infectious diseases on metabolism

\begin{tabular}{lll}
\hline & HIV infection & Other infection \\
\hline Energy expenditure & $\uparrow$ & $\uparrow$ \\
FFA turnover & $\uparrow$ & $\uparrow$ \\
Insulin sensitivity & $\uparrow$ & $\downarrow$ \\
Insulin clearance & $\uparrow$ & $\uparrow$ \\
Glucose oxidation & normal & $\downarrow$ \\
\hline
\end{tabular}


Lipid metabolism was studied after an overnight fast, 16 hrs of fasting and during short term ( $22 \mathrm{hrs}$ ) fasting [29]. After an overnight fast, fat oxidation and plasma triglyceride concentrations were significantly higher in AIDS patients, while concentrations and turnover of FFA were not significantly different between patients and controls. Short term starvation induced a more outspoken stimulation of lipolysis as FFA turnover increased to significantly higher values in the AIDS patients $(8.7 \pm 0.37 \mu \mathrm{mol} / \mathrm{kg}$ fat free mass $/ \mathrm{min})$ compared to the controls $(7.05 \mu \mathrm{mol} / \mathrm{kg}$ fat free mass $/ \mathrm{min})$.

Just as in all other above mentioned studies, these differences could not be explained by differences in the concentrations of insulin or counter-regulatory hormones.

These data in HIV infected patients are in accordance with the scarce in vitro data on metabolic effects of cytokines [2]. It is therefore quite possible that HIV infection is a good model to study metabolic effects of chronic cytokine overproduction in absence of an increased secretion of catabolic hormones.

\section{Conclusion}

When the metabolic data, after an acute TNF administration with concomitant increased plasma concentrations of the counter-regulatory hormones, are compared with those obtained during HIV infection with normal plasma values for the classical hormones, the following becomes clear (Table 3). The changes in energy expenditure and FFA turnover are in the same direction in both situations albeit at a different level; in contrast, the data on glucose metabolism are clearly opposite.

When the assumption will be proven that the metabolic changes in HIV infection are due to increased cytokine production, a few remarks can be made. It has been shown in the past that cytokines stimulate some actions of the classical hormones without having an effect itself [6]. Our data on energy expenditure and FFA turnover suggest that the possibility exists that the changes found are merely induced by a stimulation of the normal effects of the classical hormones. The data on glucose metabolism however suggest that cytokines have an unique influence on peripheral glucose uptake which will only be noticed when the

Table 3. Impact of TNF injection (with concomitant increased plasma concentrations of the classical hormones) and HIV infection (with normal plasma concentrations of these hormones) on metabolism

\begin{tabular}{lll}
\hline & TNF & HIV \\
\hline Energy expenditure & $\uparrow \uparrow$ & $\uparrow$ \\
FFA turnover & $\uparrow \uparrow$ & $\uparrow$ \\
Glucose production & $\uparrow$ & normal \\
Insulin sensitivity & $\downarrow$ & $\uparrow$ \\
Glucose oxidation & $\downarrow$ & normal \\
\hline
\end{tabular}


cytokines does not concomitantly stimulate the secretion of the classical hormones.

The exact differential roles of cytokines and classical hormones in the metabolic response to disease, as well as their interactions must be the scope of future investigations.

\section{References}

1. Beutler B, Cerami A (1987) Cachectin: more than a tumor necrosis factor. N Engl J Med 316:379-385

2. Evans RD, Argilés JM, Williamson DH (1989) Metabolic effects of tumor necrosis factoralfa (cachectin) and interleukin-1. Clin Sci 77:357-364

3. Watters JM, Wilmore DW (1989) The metabolic responses to trauma and sepsis. In: DeGroot LJ (ed) Endocrinology. Philadelphia, pp 2367-2393

4. Watters JM, Bessey PQ, Dinarello EA, Wolff SM, Wilmore DW (1986) Both inflammatory and endocrine mediators stimulate most responses to sepsis. Arch Surg 121:179-190

5. Romijn JA (1990) On metabolic modulation by starvation and disease. Thesis, University of Amsterdam

6. Warren RS, Donner DB, Starnes HF, Brennan MF (1987) Modulation of endogenous hormone action by recombinant human tumor necrosis factor. Proc Natl Acad Sci 84:8619 8622

7. Fong Y, Moldawer LL, Shires T, Lowry SF (1990) The biologic characteristics of cytokines and their implication in surgical injury. Surg Gyn Obstetr 170:363-378

8. Bendtzen $K$ (1988) Interleukin-1, interleukin-6 and tumor necrosis factor in infection, inflammation and immunity. Immunol Lett 19:183-192

9. van Deventer SJH, Büller HR, ten Cate JW, Aarden LA, Hach CE, Sturk A (1990) Experimental endotoxemia in humans: analysis of cytokine release and coagulation, fibrinolytic and complement pathways. Blood (in press)

10. Fong Y, Marano MA, Moldawer LL, et al. (1990) The acute splanchnic and peripheral tissue metabolic response to endotoxin in humans. J Clin Invest 85:1896-1904

11. Tracey KJ, Vlassara H, Cerami A (1989) Cachectin/tumor necrosis factor. Lancet 1:11221127

12. Beutler B, Mahoney J, LeTrang N, Pehala P, Cerami A (1985) Purification of cachectin, a lipoprotein lipase suppressing hormone secreted by endotoxin-induced RAW 264.7 cells. J Exp Med 161:984-995

13. Semb H, Peterson J, Tavernier J, Olivecrona T (1987) Multiple effects of tumor necrosis factor on lipoprotein lipase. J Biol Chem 262:8390-8394

14. Feingold KR, Soued M, Staprans I, et al. (1989) Effect of tumor necrosis factor (TNF) on lipid metabolism in the diabetic rat. Evidence that inhibition of adipose tissue lipoprotein lipase activity is not required for TNF-induced hyperlipidemia. J Clin Invest 83:11161121

15. Kern PA (1988) Recombinant human tumor necrosis factor does not inhibit lipoprotein lipase in primary cultures of isolated human adipocytes. J Exp Res 29:909-914

16. Vaantjes WJ, de Haas CGM, Houweling M (1990) Acute effects of interleukin 1-alfa and interleukin- 6 on intermediary metabolism in freshly isolated vat hepatocytes. Bioch Bioph Res Comm 169:623-628

17. Hill MR, Stith RD, McCallum RE (1986) Interleukin-1: a regulatory role in glucocorticoidregulated hepatic metabolism. J Immunol 137:858-862

18. Tredget EE, Yu YM, Zhong S, et al. (1988) Role of interleukin-1 and tumor necrosis factor on energy metabolism in rabbits. Am J Phys 255:E760-E768

19. Fong Y, Tracey KJ, Moldawer LL, et al. (1989) Antibodies to cachectin/tumor necrosis factor reduce interleukin-1 bèta and interleukin-6 appearance during lethal bacteremia. $J$ Exp Med 170:1627-1683 
20. Michie HR, Spriggs DR, Manogue KR, et al. (1988) Tumor necrosis factor and endotoxin induce similar metabolic responses in human beings. Surgery 104:280-286

21. Starnes HF Jr, Warren RJ, Jeevanandan M, et al. (1988) Tumor necrosis factor and the acute metabolic reponse to tissue injury in man. J Clin Invest 82:1321-1325

22. Balkwill F, Osborne R, Burke F, et al. (1987) Evidence for tumor necrosis factor/cachectin production in cancer. Lancet 2:1229-1232

23. Tracey KJ, Wei $\mathrm{H}$, Manogue KR, et al. (1988) Cachectin/tumor necrosis factor induces cachexia anemia and inflammation. J Exp Med 167:1211-1227

24. v. d. Poll T, Romijn JA, Endert E, Borm JJJ, Büller HR, Sauerwein HP (1990) Tumor necrosis factor mimics the metabolic response to acute infections in healthy humans. Clin Nutrition 9:5 (abstract)

25. Hommes MJT (1990) Host metabolism in HIV infection. Thesis, University of Amsterdam

26. Hommes MJT, Romijn JA, Godfried MH, et al. (1991) Increased resting energy expenditure in HIV-infected men. Metabolism (in press)

27. Hommes MJT, Romijn JA, Endert E, Sauerwein HP (1991) Resting energy expenditure and substrate oxidation in human immunodeficiency virus (HIV)- infected asymptomatic men. Am J Clin Nutr (in press)

28. Hommes MJT, Romijn JA, Endert E, Eeftinck-Schattenkerk JKM, Sauerwein HP (1991) Insulin sensitivity and insulin clearance in HIV infected men. Metabolism (in press)

29. Hommes MJT, Romijn JA, Endert E, Eeftinck-Schattenkerk JKM, Sauerwein HP (1991) Basal fuel hemostasis in symptomatic human immunodeficiency virus infection. Clin Science (in press) 